\title{
Bolus-to-Infusion Time in Stroke Patients Treated with Alteplase on the CT Table: A Clinical Concern
}

\author{
Ana M Iglesias Mohedano ${ }^{a} \quad$ Andrés García Pastor $^{a} \quad$ Ana de Lorenzo Pinto ${ }^{b}$ \\ Antonio C Gil Núñez ${ }^{\mathrm{a}}$ \\ aVascular Neurology Section, Stroke Center, Hospital General Universitario Gregorio Marañón, Madrid, Spain; \\ ${ }^{b}$ Department of Pharmacy, Hospital General Universitario Gregorio Marañón, Madrid, Spain
}

The onset-to-treatment time remains the most important factor in the prognosis of stroke patients treated by intravenous thrombolysis (IVT) [1]. As IVT should be administered as soon as possible, there is an increasing trend to set in-hospital targets below the initially recommended benchmark of 60 min [2].

In recent years, several measures aimed at reducing door-to-needle (DTN) times have been studied, and a degree of progress has been reported in this regard. "Ultrafast" in-hospital protocols have proven to be effective at achieving a median DTN time of $20 \mathrm{~min}$ [3].

Rapid initiation of IVT treatment on the CT table has been correlated with a shorter DTN and has in fact been recognized as the most effective measure in some studies [3, 4]. However, the sequence in which IVT treatment is carried out on CT is often not described in detail and it appears to vary considerably between different treatment centers. This could generate controversy or be cause for debate.

Although it is often reported that a "bolus of alteplase" is administered on the CT table [3,4], what is not clear is what happens with the perfusion that follows. This important topic still requires an open discussion, particularly with regard to the following issues: (1) Should IVT (bolus + perfusion) be initiated before or after CT-angiography (CTA)? (2) Should the perfusion be administered in the CT suite or can its administration be delayed

\section{KARGER}

(c) 2019 S. Karger AG, Basel

E-Mail karger@karger.com

www.karger.com/ced until arrival at the Stroke Unit? (3) How much time can elapse between the bolus and the infusion? In other words, what is the best sequence for these procedures?

In order to properly answer the above questions, it is important to focus on what we know about the pharmacokinetic of alteplase.

Pharmacokinetic data on alteplase in stroke are predominantly derived from acute myocardial infarction. In these studies, the plasma initial half-life was 3-5 min and total plasma clearance ranged from 16 to $88 \mathrm{~min}$. Alteplase is predominantly metabolized by the liver and the plasma clearance has been defined as $476-572 \mathrm{~mL} / \mathrm{min}$.

These extrapolated data from acute myocardial infarction may not be accurate due to the differences in pathophysiology and population, which is older in stroke [5]. In fact, the dose of alteplase in stroke clinical trials was not based on assessment of pharmacokinetic properties but clinical evaluation alone. In the NINDS trial, alteplase was administered as an initial 10\% IV bolus over $1 \mathrm{~min}$ followed by the continuous IV infusion of the remainder over 60 min. No further recommendations regarding the bolusinfusion interval have been specified [6]. However, as we have already pointed out, alteplase is rapidly cleared from plasma with an initial half-life of $<5 \mathrm{~min}$.

In light of the above, some studies have been conducted to simulate potential real-life situations related to rt- 
PA administration. The lesson learned is clear: delays in starting the infusion after administration of the bolus can have significant effects on serum concentrations [7]. On the other hand, a single-center series of 276 patients did not reveal significant differences in terms of independence and mortality between patients with and without bolus-infusion delay (max $8 \mathrm{~min}$ ) [8]. However, due to the small size of this study, the clinical impact of such delays remains unclear.

Based on the limited evidence, it would appear that the interval between the bolus and the infusion should be as short as possible. A number of options come to mind to avoid such delays in clinical practice: (1) CTA is performed before starting the IVT treatment (given that, according to stroke guidelines, CTA should not delay IVT [9]). There have, however, been reports that this procedure is associated with an increase in the DTN time (by as much as 13\% [10]); (2) The infusion takes place immediately after administration of the bolus and while the CTA is performed (in this case, patients should have 2 venous access sites); (3) The sequence of the treatment is bolus-CTA-infusion (provided that the bolus-infusion interval is not longer than $5 \mathrm{~min})$.

Recently, results regarding other thrombolytic agents have been presented. Tenecteplase was found to not be superior to alteplase, although it had a similar safety profile in mild stroke [11]. This new agent has a significant advantage compared with alteplase when administered as a single bolus dose. This simplicity avoids the possibility of bolus-infusion delays and could consequently reduce the variability of the serum concentration.

In conclusion, treating stroke patients in the CT suite requires rigorous planning and properly trained staff. This in-hospital measure is considered the most effective at reducing the DTN time and the perfect nexus between IVT and the selection of patients for endovascular treatment. However, achieving a balance between safety and speed is a very important issue, and in-hospital protocols should be designed that take into account the pharmacokinetic characteristics of alteplase. Until the use of the latest thrombolytic drugs becomes commonplace, the "DTN" time with alteplase appears to be equally important as the "door-to-infusion" time. Consequently, both intervals should be carefully monitored.

\section{Disclosure Statement}

The authors declare that they have no conflicts of interest to disclose.

\section{Funding Sources}

None.

\section{Author Contributions}

All authors have contributed equally to this work.

\section{References}

1 Emberson J, Lees KR, Lyden P, Blackwell L, Albers G, Bluhmki E, et al.; Stroke Thrombolysis Trialists' Collaborative Group. Effect of treatment delay, age, and stroke severity on the effects of intravenous thrombolysis with alteplase for acute ischaemic stroke: a meta-analysis of individual patient data from randomised trials. Lancet. 2014 Nov;384(9958):1929-35.

2 Kamal N, Benavente O, Boyle K, Buck B, Butcher K, Casaubon LK, et al. Good is not Good Enough: The Benchmark Stroke Doorto-Needle Time Should be 30 Minutes. Can J Neurol Sci. 2014 Nov;41(6):694-6.

3 Kamal N, Smith EE, Jeerakathil T, Hill MD. Thrombolysis: improving door-to-needle times for ischemic stroke treatment - A narrative review. Int J Stroke. 2018 Apr;13(3): 268-76.

4 Iglesias Mohedano AM, García Pastor A, Díaz Otero F, Vázquez Alen P, Martín Gómez MA, Simón Campo P, et al. A new protocol reduces median door-to-needle time to the benchmark of 30 minutes in acute stroke treatment. Neurologia. 2018;pii:S0213-4853(18)30119-1.

5 Acheampong P, Ford GA. Pharmacokinetics of alteplase in the treatment of ischaemic stroke. Expert Opin Drug Metab Toxicol. 2012 Feb;8(2):271-81.

6 National Institute of Neurological Disorders and Stroke rt-PA Stroke Study Group. Tissue plasminogen activator for acute ischemic stroke. N Engl J Med. 1995;333:1581-7.

7 Smith C, Al-Nuaimi Y, Wainwright J, Sherrington C, Singh A, Kallingal J, et al. The influence of bolus to infusion delays on plasma Tissue Plasminogen Activator levels. Int J Stroke. 2014 Oct;9(7):939-42.

8 Acheampong P, May MT, Ford GA, Dixit AK. Bolus-infusion delays of alteplase during thrombolysis in acute ischaemic stroke and functional outcome at 3 months. Stroke Res Treat. 2014;2014:358640.
9 Powers WJ, Rabinstein AA, Ackerson T, Adeoye OM, Bambakidis NC, Becker K, et al.; American Heart Association Stroke Council. 2018 Guidelines for the Early Management of Patients With Acute Ischemic Stroke: A Guideline for Healthcare Professionals From the American Heart Association/American Stroke Association. Stroke. 2018 Mar;49(3):e46-110.

10 García Pastor A, Díaz Otero F, Gil Navarro S, Pablo Cuello J, Sobrino García P, García Arratibel $\mathrm{A}$, et al. Vascular imaging before intravenous thrombolysis: consequences of inhospital delay in applying two diagnostic procedures. J Neuroimaging. 2015 May-Jun; 25(3):397-402.

11 Logallo N, Novotny V, Assmus J, Kvistad CE, Alteheld L, Rønning OM, et al. Tenecteplase versus alteplase for management of acute ischaemic stroke (NOR-TEST): a phase 3, randomised, open-label, blinded endpoint trial. Lancet Neurol. 2017 Oct;16(10):781-8. 